\title{
Searching for Possible Members of Star Moving Groups in the Kepler Field
}

\author{
Chang Yao Chen ${ }^{1}$ W. P. Chen ${ }^{1,2}$ and Ali Luo ${ }^{3}$ \\ ${ }^{1}$ Department of Physics, National Central University, \\ 300 Zhongda Rd., Zhongli, Taoyuan 32001, Taiwan \\ email: a.e.johnny@hotmail.com \\ ${ }^{2}$ Institute of Astronomy, National Central University, Taiwan \\ ${ }^{3}$ National Astronomical Observatories, Chinese Academy of Sciences, Beijing, 100012, China
}

\begin{abstract}
We present identification of candidate members of the Beta Pictoris, AB Doradus, and TW Hydrae moving star groups. Our pilot study toward the Kepler field, where a multitude of stellar parameters are available, led to a list of 19 highly probable candidates that share the same range of distance and space (tangential and radial) motion as known members in a moving group. A few candidates lack radial velocity data, but are ROSAT X-ray sources, so are considered as likely young stars.
\end{abstract}

Keywords. star clusters and associations, kinematics

\section{Moving Star Groups}

It is known that stars are formed in groups out of molecular clouds, with dozens to thousands of members. These clusters dissociate with time (Zuckerman et al. 2001), but the then-members, when young, still share the same motion through space. There are eight known moving groups of stars, all young $(\sim 10-100 \mathrm{Myr})$ and nearby (within $120 \mathrm{pc}$ ) (Torres et al. 2008). Interestingly, most groups have known members scattered preferentially in the southern sky. It is unknown whether such a skew distribution is intrinsic, e.g., by Galactic tidal distortion, or a result of selection bias.

As a pilot program to identify candidate members of moving groups, we focused on the Kepler field in the northern sky to take advantage of the wealth of stellar data to search for possible members of the Beta Pictoris, AB Doradus, and TW Hydrae moving groups. The $\epsilon$ Chamaeleontis group is away from the line of sight to the Kepler field (see Fig. 1), so its candidate list serves to assess the false positive rate of our selection.

\section{Possible Members}

We searched in a $16 \times 16 \mathrm{deg}^{2}$ region centering on the Kepler field, at R.A.=290.7 deg, and decl. $=44.5 \mathrm{deg}(\mathrm{J} 2000)$, in which there are some 1.2 million UCAC4 objects. Using the space location and motion of known members of a particular moving group, we computed the expected proper motion and the radial velocity of a member in the entire sky. Fig. 2 shows the example for the Beta Pic group. The stellar distance was estimated by the 2MASS $K$ magnitude and $J-H$ color, assuming it is a dwarf (Cox 2000). No extinction was considered. A candidate is identified if its space tangential velocity, computed by its distance and proper motion, is within the range of those of known members.

While thousands of sources satisfied the tangential velocity plus distance criteria, about $1 / 5$ of these can be further winnowed by the radial velocity data by LAMOST (Large Sky Area Multi-Object Fiber Spectroscopic Telescope). A total of 121 stars (34 of Beta 


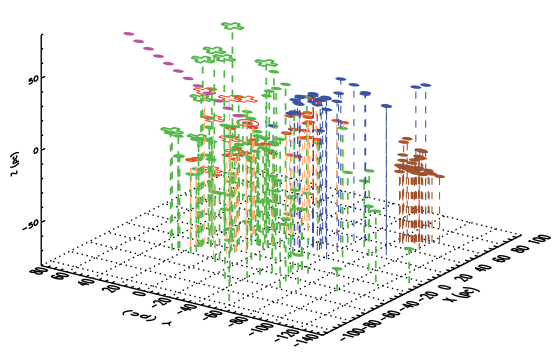

Figure 1. Spatial positions of known members of the Beta Pic (orange), AB Dor (green), TW Hyd (blue), and $\epsilon$ Cha (brown) moving groups (Torres et al. 2008). The cross symbols are members from Schlieder et al. 2012. Sun's location is at origin. Pink dots illustrates the line of sight toward the Kepler field.

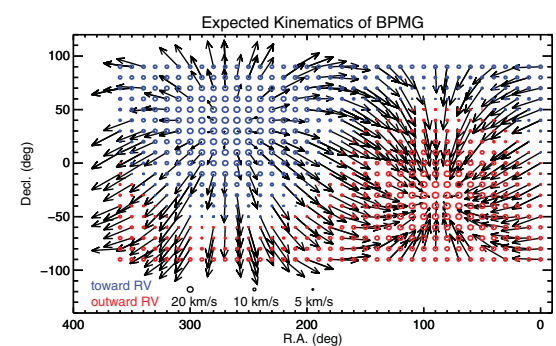

Figure 2. The expected space motion of the Beta Pic moving group on the whole sky in equatorial coordinates. The proper motions are shown as black arrows, whereas the radial velocities are depicted as color circles (blue or red shifted)

Pic, 76 of AB Dor, and 11 of TW Hyd groups) have distances, proper motions, and radial velocities consistent with being possible members. Among these, only 19 have been observed at multiple epochs and show no obvious variations caused by binary orbits; these are hence probable members. There are also a few ROSAT X-Ray sources (Voges et al. 1999), signifying stellar youth or activity, that lack radial velocity measurements. More spectroscopic radial velocity data are being collected.

The false positive rate was estimated based on the 121 candidates. For each group, the corresponding number density is, respectively, $1.6 \times 10^{-3} \mathrm{pc}^{-3}$ for the Beta Pic group, $3.5 \times 10^{-3} \mathrm{pc}^{-3}$ for AB Dor, and $1.5 \times 10^{-3} \mathrm{pc}^{-3}$ for TW Hyd. In comparison, the number density toward the $\epsilon$ Cha group is $1.5 \times 10^{-3} \mathrm{pc}^{-3}$. This suggests that most candidates should be simply chance field stars. Additional diagnosis of membership includes, other than X-ray emission, stellar flares in the (Kepler) light curve, lithium absorption in the spectrum, or infrared excess arising from a circumstellar debris disk.

We acknowledge the grant by the Ministry of Science and Technology MOST103-2112M-008-024-MY3. We also thank the LAMOST staff for help with the LAMOST data.

\section{References}

Cox, A. N. 2000, Allen's Astrophysical Quantities, 4th ed., Am. Inst. Phys., AIP Press, SpringerVerlag, New York

Schlieder, J. E., Lépine, S., \& Simon, M. 2012, AJ, 144, 109

Torres, C. A. O., Quast, G. R., Melo, C. H. F., \& Sterzik, M. F. 2008, in Handbook of Star Forming Regions, Volume II, Reipurth B., ed., p. 757

Voges, W., et al. 1999, A\&\&A, 349, 389

Zuckerman, B., Song, I., Bessell, M. S., \& Webb, R. A. 2001, The ApJL, 562, L87 